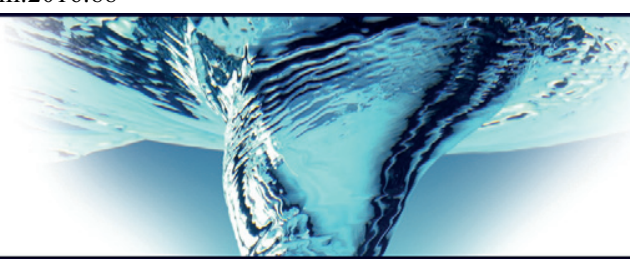

\title{
Analytical formulae for longitudinal slip lengths over unidirectional superhydrophobic surfaces with curved menisci
}

\author{
Darren G. Crowdy $\dagger$ \\ Department of Mathematics, Imperial College London, 180 Queen's Gate, London SW7 2AZ, UK \\ (Received 12 November 2015; revised 29 December 2015; accepted 30 January 2016; \\ first published online 24 February 2016)
}

This paper reports new analytical formulae for the longitudinal slip lengths for simple shear over a superhydrophobic surface, or bubble mattress, comprising a periodic array of unidirectional circular menisci, or bubbles, protruding into, or out of, the fluid. The accuracy of the formulae is tested against results from full numerical simulations; they are found to give small relative errors even at large no-shear fractions. In the dilute limit the formulae reduce to an earlier result by Crowdy (Phys. Fluids, vol. 22, 2010, 121703). They also extend analytical results of Sbragaglia \& Prosperetti (Phys. Fluids, vol. 19, 2007, 043603) beyond the limit of a small protrusion angle.

Key words: drag reduction, interfacial flows, superhydrophobic surface

\section{Introduction}

Superhydrophobic surfaces are typically no-slip surfaces endowed with additional microstructural features such as grooves, posts or holes and they can significantly reduce fluid drag in microchannels (Rothstein 2010). In many cases, in the Cassie state the microstructural surface features are occupied by pockets of gas rather than being liquid-filled (as in the Wenzel state). The spanning gas-liquid interfaces, or menisci, that can protrude into or out of the fluid are then often close to being shear-free and allow slip. The drag reduction characteristics of such a surface have a strong dependence on the geometrical properties of these gas-liquid interfaces (Steinberger et al. 2007) and it is an important matter to be able to quantify them. For shear flows with shear rate $\dot{\gamma}$ over such a surface occupying the plane $y=0$ the velocity field far from the plane of the surface takes the form

$$
\boldsymbol{u}=\dot{\gamma}(y+\lambda) \hat{\boldsymbol{x}}
$$

$\dagger$ Email address for correspondence: d.crowdy@imperial.ac.uk 
(a)

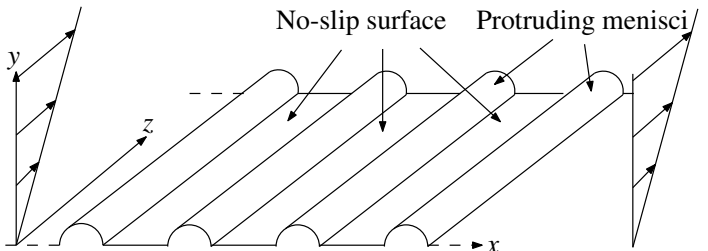

(b)

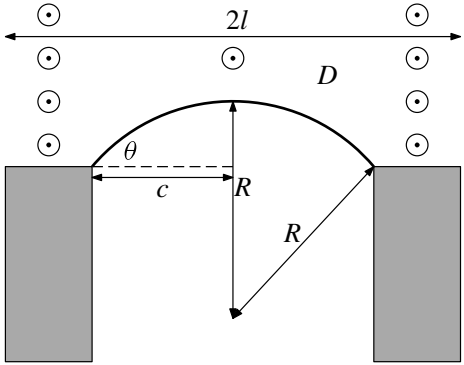

FIGURE 1. Longitudinal shear flow over a surface comprising a unidirectional periodic array, with period $2 l$, of menisci of width $2 c$ protruding at angle $\theta$ with intermediate noslip zones.

where $\hat{\boldsymbol{x}}$ is the flow direction. The constant $\lambda$ is the effective slip length and is a measure of the frictional properties of the surface: it is the fictional distance below the surface at which the shear flow would extrapolate to zero.

In applications an important class of superhydrophobic surfaces comprises those with a periodic array of grooves aligned with the principal flow direction. Figure 1 shows a schematic diagram of such a surface in the Cassie state when a periodic array of parallel circular menisci of width $2 c$ and period $2 l$, each having protrusion angle $\theta$, span unidirectional grooves aligned along a $z$-axis, say, with no-slip regions in between. Also shown is the period window $-l<x<l$ with the meniscus lying in the subinterval $-c<x<c$. An important geometrical characteristic of such a 'bubble mattress' is the no-shear fraction $c / l$. When no-shear conditions are imposed on the menisci a complete analytical characterization of the effective slip properties of such surfaces is known in the dilute limit of small no-shear fraction. Davis \& Lauga (2009) derived an explicit formula for transverse slip length over such a surface; Crowdy (2010) derived the corresponding result for longitudinal slip length. These results are valuable together because, for small no-shear fractions and zero capillary number, the slip length for a linear shear in a general direction over the surface is a linear combination of these results.

In other analytical work, Sbragaglia \& Prosperetti (2007) used a boundary perturbation analysis to investigate the effects of small interface curvature/protrusion on the effective slip length in a pressure-driven flow in channels. Their analysis is valid for any no-shear fraction, but it is limited to small protrusion angles of the menisci (close to flat). Numerical calculations of the slip lengths associated with such bubble mattresses have been performed by Teo \& Khoo (2010) and $\mathrm{Ng}$ \& Wang (2011).

The present paper extends the author's earlier result (Crowdy 2010) beyond the dilute limit and produces formulae for the longitudinal slip length over these unidirectional surfaces that are accurate over a much larger range of no-shear fractions. Crowdy (2010) derived the longitudinal slip length $\lambda_{0}$ in the dilute limit as

$$
\frac{\lambda_{0}}{c}=c \delta \alpha(\theta), \quad \alpha(\theta)=\frac{3 \pi^{2}-4 \pi \theta+2 \theta^{2}}{6(\pi-\theta)^{2}}, \quad \delta \equiv \frac{\pi}{2 l} .
$$


The first of two new results of the present paper is to derive the more accurate formula:

$$
\frac{\lambda_{1}}{c}=\frac{c \delta \alpha(\theta)}{\left[1-\frac{(c \delta)^{2} \alpha(\theta)}{3}\right]}
$$

where we use $\lambda_{1}$ to denote the improved result. We also write down the associated flow field. For no-shear fractions as high as $c / l=0.75$ (1.3) gives agreement with maximum relative errors (across protrusion angles) of 6-7\% compared to the numerical results of Teo \& Khoo (2010). Clearly (1.3) reduces to $(1.2 a-c)$ to leading order in $c \delta$ (or, equivalently, to leading order in the no-shear fraction $c / l$ ). We have not presented (1.3) as an expansion in powers of $c / l$ because the functional form (1.3) arises naturally in our analysis and it turns out to give more accurate results than a truncated formal expansion.

The second result is to derive an even higher-order approximation, denoted by $\lambda_{2}$, reported later in (4.13). It is not as simple to write down as (1.3), but it is nonetheless explicit and reduces the maximum relative errors in the slip length to as little as $1-2 \%$ for no-shear fraction $c / l=0.75$; even for a no-shear fraction as high as $c / l=0.9$, it is still accurate to within a maximum relative error of $8-9 \%$ (see figure 3). Moreover, an approximation of the higher-order formula (4.13) (which ignores certain terms in (4.13) that are sixth order in the no-shear fraction) is the more concise, and only slightly less accurate, expression

$$
\frac{\lambda_{2}}{c} \approx \frac{c \delta \alpha(\theta)\left[1+\frac{(c \delta)^{4} \beta(\theta)}{15}\right]}{\left[1-\frac{(c \delta)^{2} \alpha(\theta)}{3}-\frac{(c \delta)^{4} \beta(\theta)}{15}\right]},
$$

with

$$
\beta(\theta)=\frac{1}{360(\pi-\theta)^{4}}\left(32 \theta^{4}-128 \pi \theta^{3}+212 \pi^{2} \theta^{2}-168 \pi^{3} \theta+45 \pi^{4}\right) .
$$

This formula gives the required slip length with maximum relative error $2 \%$ for noshear fractions in the range $c / l \in[0,0.75]$ and $12 \%$ for $c / l=0.9$.

Formulae (1.3), (4.13) and (1.4) are the main results of this paper. They are valid without restriction on the protrusion angle $\theta$. We show that they extend an analytical result of Sbragaglia \& Prosperetti (2007) beyond the limit of small protrusion angle. The formulae can, in principle, be derived using the usual formalisms of matched asymptotic expansions but we derive them using our own mathematical approach, which is of theoretical interest in itself.

\section{The dilute limit}

Crowdy (2010) has previously derived the formula $(1.2 a-c)$ for the longitudinal slip length relevant in the dilute limit $c / l \rightarrow 0$; it is natural to think of fixing $c=1$, say, and taking $l \rightarrow \infty$. Those prior results can be summarized as follows. With $z=x+\mathrm{i} y$ we consider a complex potential $h_{s}(z)$, analytic in the fluid region, such that

$$
w(x, y)=\dot{\gamma} \operatorname{Im}\left[h_{s}(z)\right]
$$




\section{G. Crowdy}

The imposed far-field condition of simple shear requires that $w(x, y) \sim \dot{\gamma} y$, hence

$$
h_{s}(z) \sim z+O(1 / z), \quad \text { as } z \rightarrow \infty .
$$

To satisfy the no-slip condition on the wall we need

$$
\operatorname{Im}\left[h_{s}(z)\right]=0, \quad \text { on } y=0, x \notin[-c, c],
$$

while the no-stress condition on the meniscus requires that

$$
\operatorname{Re}\left[h_{s}(z)\right]=0, \quad \text { on the meniscus, }
$$

which follows from the condition $\partial w / \partial n=0$ on use of the Cauchy-Riemann equations.

To solve this problem Crowdy (2010) employed conformal mapping techniques. With $\beta=\pi-\theta$ the conformal mapping transplanting the upper half of the unit disc in a parametric complex $\zeta$-plane to the fluid region, and its inverse mapping function, are

$$
z(\zeta)=c\left[\frac{(1-\zeta)^{2 \beta / \pi}+(1+\zeta)^{2 \beta / \pi}}{(1-\zeta)^{2 \beta / \pi}-(1+\zeta)^{2 \beta / \pi}}\right], \quad \zeta(z)=\frac{(z / c-1)^{\pi / 2 \beta}-(z / c+1)^{\pi / 2 \beta}}{(z / c-1)^{\pi / 2 \beta}+(z / c+1)^{\pi / 2 \beta}} .
$$

Having derived these, Crowdy (2010) uses them to establish that

$$
h_{s}(z)=-\frac{2 \pi c}{\beta}\left[\frac{\left((z / c)^{2}-1\right)^{\pi / 2 \beta}}{(z / c-1)^{\pi / \beta}-(z / c+1)^{\pi / \beta}}\right] \sim z+\frac{\Delta_{1}}{z}+\frac{\Delta_{3}}{z^{3}}+\cdots, \quad \text { as }|z| \rightarrow \infty,
$$

where

$$
a=\frac{\pi}{2(\pi-\theta)}, \quad \Delta_{1}=-\frac{c^{2}}{3}\left(2 a^{2}+1\right), \quad \Delta_{3}=-\frac{2 c^{4}}{45}\left(-7 a^{4}+5 a^{2}+2\right) . \quad(2.7 a-c)
$$

Actually, only $\Delta_{1}$ is needed to derive $(1.2 a-c)$ based on a simple superposition argument.

Since his focus was to find the slip length Crowdy (2010) did not report the corresponding dilute-limit complex potential, but it will be useful for what follows. It turns out to be

$$
h_{0}(z)=h_{s}(z)-\frac{\Delta_{1}}{z}-\lambda_{0} \cot (\delta z), \quad-\frac{\lambda_{0}}{\delta}=\Delta_{1} .
$$

We now explain this result because it helps to understand our derivations of the higherorder approximations. If $h(z)$ denotes the exact solution to the periodic problem then $h(z)$ must be analytic in the period window $D$ shown in figure 1 and satisfy the quasiperiodicity condition

$$
h(z+2 l)=h(z)+2 l
$$

in order that the velocity $w=\dot{\gamma} \operatorname{Im}[h(z)]$ is periodic; the quasi-periodicity here is induced by the far-field $z$ behaviour. Similarly, $h_{0}(z)$ is required to be analytic in $D$. This is true of $h_{0}(z)$ given in (2.8) since $h_{s}(z)$ is analytic there - it is analytic, by construction, everywhere in the upper half-plane and above the meniscus - while the apparent singularity of $h_{0}(z)$ at $z=0$, which is inside the period window if $\theta<0$, is 


\section{Analytical formulae for longitudinal slip lengths}

in any case removable by the choice of $\lambda_{0}$. Moreover, the cotangent function has a periodic array of singularities along the real axis but the two closest to the one at $z=0$ are in the two period windows neighbouring $D$ and not inside $D$ itself.

The first term added to $h_{s}(z)$ in (2.8) serves the purpose of removing the $O(1 / z)$ behaviour of $h_{s}(z)$ as $z \rightarrow \infty$ so that, as $z \rightarrow \infty$,

$$
h_{0}(z) \sim z+\mathrm{i} \lambda_{0}+O\left(1 / z^{3}\right),
$$

where we have used the fact that $\cot (\delta z) \rightarrow-\mathrm{i}$ as $y \rightarrow+\infty$ and the known far-field asymptotics (2.6) of $h_{s}(z)$. This means that, on the edges of the period window where $|z| \geqslant l$, the function $h_{0}(z)$ satisfies

$$
h_{0}(z+2 l)=h_{0}(z)+2 l+O\left(\delta^{3}\right),
$$

which is a good approximation to the quasi-periodicity condition (2.9) if $\delta$ is small. It is easily checked that $h_{0}(z)$ is real when $z$ is real since this is true of $h_{s}(z)$, implying that $h_{0}(z)$ satisfies the no-slip condition on the wall. Also, provided $\delta$ is small, then on the meniscus

$$
\begin{aligned}
\operatorname{Re}\left[h_{0}(z)\right] & =\operatorname{Re}\left[h_{s}(z)-\frac{\Delta_{1}}{z}-\lambda_{0} \cot (\delta z)\right] \\
& \sim \operatorname{Re}\left[-\frac{\Delta_{1}}{z}-\lambda_{0}\left\{\frac{1}{\delta z}-\frac{\delta z}{3}+\cdots\right\}\right]=\operatorname{Re}\left[\frac{\lambda_{0} \delta z}{3}+\cdots\right] \\
& =O\left(\lambda_{0} c \delta\right)=O\left(c^{2} \delta^{2}\right) .
\end{aligned}
$$

Thus $h_{0}(z)$ also satisfies the meniscus boundary condition correct to $O\left(c^{2} \delta^{2}\right)$. In this way we have verified that $h_{0}(z)$ is the approximation to the required complex potential provided that $\delta$ is small.

\section{An improved slip length formula}

To produce an improved formula for the slip length, accurate at even larger noshear fractions, it is natural to seek a higher-order approximation in $\delta c$. Consider the modified complex potential

$$
h_{1}(z)=h_{s}(z)+\frac{\lambda_{1}}{\delta} \frac{1}{z}-\lambda_{1} \cot (\delta z)+\frac{\lambda_{1} \delta}{3}\left[h_{s}(z)-z\right],
$$

with $\lambda_{1}$ now chosen to satisfy

$$
\Delta_{1}+\frac{\lambda_{1}}{\delta}+\frac{\lambda_{1} \delta \Delta_{1}}{3}=0 .
$$

With this choice the $1 / z$ term in the far-field expansion of the three non-cot terms

$$
h_{s}(z), \quad+\frac{\lambda_{1}}{\delta} \frac{1}{z}, \quad+\frac{\lambda_{1} \delta}{3}\left[h_{s}(z)-z\right]
$$

of $h_{1}(z)$ vanishes. This ensures that, as $z \rightarrow \infty$,

$$
h_{1}(z) \sim z+\mathrm{i} \lambda_{1}+O\left(1 / z^{3}\right),
$$




\section{G. Crowdy}

where we have again used the fact that $\cot (\delta z) \rightarrow-\mathrm{i}$ as $y \rightarrow+\infty$ and the known far-field asymptotics (2.6) of $h_{s}(z)$. Hence $h_{1}(z)$ has the correct far-field behaviour with slip length $\lambda_{1}$. On the edges of the period window where $|z| \geqslant l$, we still have

$$
h_{1}(z+2 l)=h_{1}(z)+2 l+O\left(\delta^{3}\right)
$$

which is a good approximation to the required quasi-periodicity (2.9) if $\delta$ is small. The apparent singularity of the function (3.1) at $z=0$ is again removable while the nearest other singularities of the cotangent function are in the neighbouring period windows as before. It is therefore confirmed that $h_{1}(z)$ is analytic in $D$. When $z$ is real, which is true on the no-slip wall, so is $h_{1}(z)$, confirming that the no-slip condition is satisfied there. Finally, on expansion of the cotangent for small $\delta$, notice that on the meniscus,

$$
\begin{aligned}
\operatorname{Re}\left[h_{1}(z)\right] & =\operatorname{Re}\left[h_{s}(z)+\frac{\lambda_{1}}{\delta} \frac{1}{z}-\lambda_{1} \cot (\delta z)+\frac{\lambda_{1} \delta}{3}\left[h_{s}(z)-z\right]\right] \\
& \sim \operatorname{Re}\left[+\frac{\lambda_{1}}{\delta} \frac{1}{z}-\lambda_{1}\left\{\frac{1}{\delta z}-\frac{\delta z}{3}+O\left(c^{3} \delta^{3}\right)\right\}-\frac{\lambda_{1} \delta z}{3}\right] \\
& =O\left(\lambda_{1} c^{3} \delta^{3}\right)=O\left(c^{4} \delta^{4}\right),
\end{aligned}
$$

where we have used (2.4) twice as well as the Laurent expansion of $\cot (\delta z)$ about $z=0$. Hence, (3.1) satisfies the boundary conditions on the meniscus, the no-slip wall and the edges of the period window correct to $O\left(c^{3} \delta^{3}\right)$. The quantity $\lambda_{1}$ as given by (3.2) is now the required slip length at this order of approximation and produces the result (1.3). Teo \& Khoo (2010) report the slip lengths $\lambda_{T K}$, say, with the renormalizations

$$
\lambda_{T K}=\frac{\lambda_{1}}{2 / l}=\frac{\lambda_{1}}{4 \delta / \pi}
$$

where we have taken $c=1$. Figure 2 shows $\lambda_{T K}$, as a function of protrusion angle $\theta$, for $c / l=0.1,0.25,0.5,0.75$ and 0.9 together with corresponding data points from Teo \& Khoo (2010), which serve as our benchmark solution. The maximum relative error of this approximation for $c / l=0.75$ is between 6 and $7 \%$ and for $c / l=0.9$ is around $25 \%$.

\section{Higher-order analysis}

The analysis of the previous section is readily extended to even higher order. We introduce $\tilde{h}_{s}(z)$ as the complex potential for a single protruding bubble with all the same boundary conditions as for $h_{s}(z)$ but now satisfying the modified far-field condition

$$
\tilde{h}_{s}(z) \sim z^{3}+O(1 / z), \quad \text { as } z \rightarrow \infty
$$

This far-field flow is no longer a simple shear. By the same conformal mapping arguments (Crowdy 2010) used to find $h_{s}(z)$ it can be shown that

$$
\tilde{h}_{s}(z)=-a^{3} c^{3}\left[\frac{1}{\zeta^{3}}-\zeta^{3}\right]+c^{2}\left(1-a^{2}\right)\left[\frac{1}{\zeta}-\zeta\right] \sim z^{3}+\frac{\tilde{\Delta}_{1}}{z}+\frac{\tilde{\Delta}_{3}}{z^{3}}+\cdots, \quad \text { as }|z| \rightarrow \infty,
$$



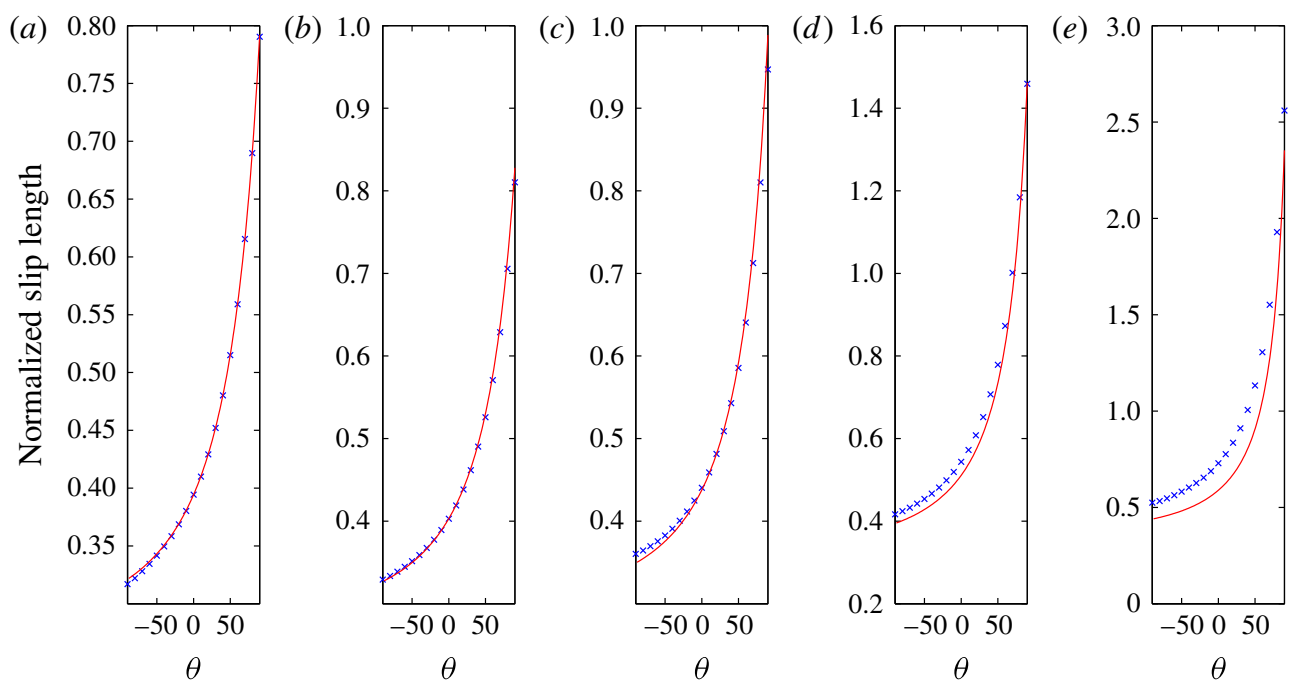

FIGURE 2. Normalized slip length (3.7) as a function of protrusion angle $\theta$ for $c / l=0.1$ $(a), 0.25(b), 0.5(c), 0.75(d)$ and $0.9(e)$. Crosses show numerical data from Teo \& Khoo (2010).

where we have used (2.5) and

$$
\left.\begin{array}{c}
\tilde{\Delta}_{1}=-\frac{2 c^{4}}{15}\left(-7 a^{4}+5 a^{2}+2\right), \\
\tilde{\Delta}_{3}=-\frac{c^{6}}{3780}\left(4855 a^{6}+945 a^{5}-5817 a^{4}+6615 a^{3}-6090 a^{2}+3780 a-508\right) .
\end{array}\right\}
$$

Now consider the new complex potential, constructed using $h_{s}(z)$ and $\tilde{h}_{s}(z)$, given by

$$
\begin{aligned}
h_{2}(z)= & h_{s}(z)+\frac{\lambda_{2}}{\delta} \frac{1}{z}+\frac{\mu_{2}}{\delta^{3}} \frac{1}{z^{3}}-\lambda_{2} \cot (\delta z)-\mu_{2} \cot (\delta z) \operatorname{cosec}^{2}(\delta z) \\
& +\left[\frac{1}{3} \lambda_{2} \delta+\frac{1}{15} \mu_{2} \delta\right]\left(h_{s}(z)-z\right)+\left[\frac{1}{45} \lambda_{2} \delta^{3}+\frac{20}{945} \mu_{2} \delta^{3}\right]\left(\tilde{h}_{s}(z)-z^{3}\right),
\end{aligned}
$$

where the real parameters $\lambda_{2}$ and $\mu_{2}$ are chosen to satisfy

$$
\left.\begin{array}{l}
\Delta_{1}+\frac{\lambda_{2}}{\delta}+\left[\frac{1}{3} \lambda_{2} \delta+\frac{1}{15} \mu_{2} \delta\right] \Delta_{1}+\left[\frac{1}{45} \lambda_{2} \delta^{3}+\frac{20}{945} \mu_{2} \delta^{3}\right] \\
\tilde{\Delta}_{1}=0, \\
\Delta_{3}+\frac{\mu_{2}}{\delta^{3}}+\left[\frac{1}{3} \lambda_{2} \delta+\frac{1}{15} \mu_{2} \delta\right] \Delta_{3}+\left[\frac{1}{45} \lambda_{2} \delta^{3}+\frac{20}{945} \mu_{2} \delta^{3}\right] \tilde{\Delta}_{3}=0 .
\end{array}\right\}
$$

We claim that $h_{2}(z)$ is the required higher-order solution. To see this, the expansions

$$
\cot z=\frac{1}{z}-\frac{z}{3}-\frac{z^{3}}{45}-\frac{2}{945} z^{5}+\cdots, \quad \cot (z) \operatorname{cosec}^{2}(z)=\frac{1}{z^{3}}-\frac{z}{15}-\frac{20}{945} z^{3}+\cdots,
$$




\section{G. Crowdy}

reveal that the third-order singularity of $h_{2}(z)$ at $z=0$ is removable. Furthermore,

$$
h_{2}(z) \rightarrow z+\mathrm{i} \lambda_{2}+O\left(1 / z^{5}\right)
$$

where we have used the facts that $\cot (\delta z) \rightarrow-\mathrm{i}$ and $\cot (\delta z) \operatorname{cosec}^{2}(\delta z)$ decays exponentially as $y \rightarrow+\infty$, as well as the far-field forms (2.6) and (4.2) of $h_{s}(z)$ and $\tilde{h}_{s}(z)$. Then on the edges of the period window where $|z| \geqslant l$,

$$
h_{2}(z+2 l)=h_{2}(z)+2 l+O\left(\delta^{5}\right)
$$

so that $h_{2}(z)$ satisfies the required quasi-periodicity condition there (now to higher order than before). $\lambda_{2}$ is the required slip length. For $z$ on the no-slip wall $h_{2}(z)$ is real, implying that the no-slip condition is satisfied there. On expanding (4.4) for small $\delta$, and on use of (4.5),

$$
\begin{aligned}
\operatorname{Re}\left[h_{2}(z)\right]= & \operatorname{Re}\left[\frac{\lambda_{2}}{\delta} \frac{1}{z}+\frac{\mu_{2}}{\delta^{3}} \frac{1}{z^{3}}-\lambda_{2}\left[\frac{1}{\delta z}-\frac{\delta z}{3}-\frac{\delta^{3} z^{3}}{45}+O\left(\delta^{5} c^{5}\right)\right]\right. \\
& -\mu_{2}\left[\frac{1}{\delta^{3} z^{3}}-\frac{\delta z}{15}-\frac{20}{945} \delta^{3} z^{3}+O\left(\delta^{5} c^{5}\right)\right]-\left[\frac{1}{3} \lambda_{2} \delta+\frac{1}{15} \mu_{2} \delta\right] z \\
& \left.-\left[\frac{1}{45} \lambda_{2} \delta^{3}+\frac{20}{945} \mu_{2} \delta^{3}\right] z^{3}\right]=O\left(\lambda_{1} c^{5} \delta^{5}\right)
\end{aligned}
$$

where we have used the Laurent expansions (4.6) and the fact that

$$
\operatorname{Re}\left[h_{s}(z)\right]=\operatorname{Re}\left[\tilde{h}_{s}(z)\right]=0, \quad \text { on the meniscus. }
$$

Hence the meniscus boundary condition is satisfied correct to $O\left(c^{6} \delta^{6}\right)$. The solution of the $2 \times 2$ system (4.5) gives the required slip length $\lambda_{2}$. In matrix form it is

$$
\left(\begin{array}{ll}
a_{11} & a_{12} \\
a_{21} & a_{22}
\end{array}\right)\left(\begin{array}{l}
\lambda_{2} \\
\mu_{2}
\end{array}\right)=\left(\begin{array}{c}
-\Delta_{1} \delta \\
-\Delta_{3} \delta^{3}
\end{array}\right),
$$

where

$$
\left.\begin{array}{l}
a_{11}=1+\frac{\Delta_{1} \delta^{2}}{3}+\frac{\tilde{\Delta}_{1} \delta^{4}}{45}, \quad a_{12}=\frac{\Delta_{1} \delta^{2}}{15}+\frac{20 \tilde{\Delta}_{1} \delta^{4}}{945}, \\
a_{21}=\frac{\Delta_{3} \delta^{4}}{3}+\frac{\tilde{\Delta}_{3} \delta^{6}}{45}, \quad a_{22}=1+\frac{\Delta_{3} \delta^{4}}{15}+\frac{20 \tilde{\Delta}_{3} \delta^{6}}{945} .
\end{array}\right\}
$$

In view of (2.7) and (4.3) all matrix elements are known as explicit functions of $a$ and $c$, consequently an explicit formula for the slip length $\lambda_{2}$ is

$$
\lambda_{2}=\frac{\Delta_{3} \delta^{3} a_{12}-\Delta_{1} \delta a_{22}}{a_{11} a_{22}-a_{12} a_{21}} .
$$

The normalized slip length (3.7) - but now with $\lambda_{1}$ replaced by $\lambda_{2}$ as given by (4.13) - is plotted in figure 3 , as a function of $\theta$ for $c / l=0.1,0.25,0.5,0.75$ and 0.9 . It gives markedly better agreement with the numerical solution at $c / l=0.9$ than that shown in figure 2. The maximum relative error of this approximation for $c / l=0.75$ is between 1 and $2 \%$, and for $c / l=0.9$ it is between 8 and $9 \%$. 

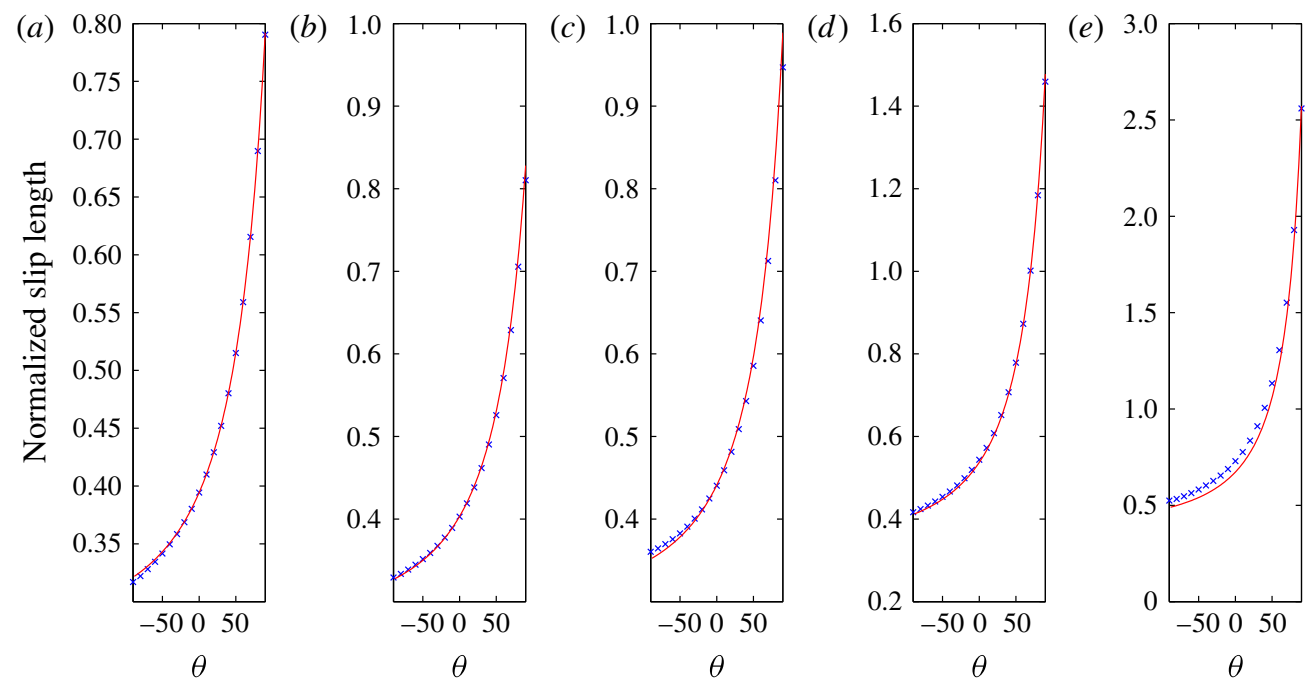

FIGURE 3. Normalized slip length, now based on the approximation $\lambda_{2}$ in (4.13), as a function of $\theta$ for $c / l=0.1(a), 0.25(b), 0.5(c), 0.75(d)$ and $0.9(e)$. Crosses show numerical data from Teo \& Khoo (2010).

Our analysis leads naturally to formula (4.13) but if we use the second equation in (4.5) to approximate

$$
\mu_{2} \approx-\Delta_{3} \delta^{3}
$$

then, on substitution of this into the first equation in (4.5), we find the approximation

$$
\lambda_{2} \approx-\frac{\delta \Delta_{1}\left(1-\frac{\delta^{4} \Delta_{3}}{15}\right)}{1+\frac{\Delta_{1} \delta^{2}}{3}+\frac{\delta^{4} \Delta_{3}}{15}} .
$$

On eliminating $a$ in favour of $\theta$, it can be shown that

$$
\left.\begin{array}{c}
\Delta_{1}=-\frac{c^{2}}{6(\pi-\theta)^{2}}\left(2 \theta^{2}-4 \pi \theta+3 \pi^{2}\right), \\
c^{4} \\
\left(32 \theta^{4}-128 \pi \theta^{3}+212 \pi^{2} \theta^{2}-168 \pi^{3} \theta+45 \pi^{4}\right) .
\end{array}\right\}
$$

Equation (4.15) is now equivalent to (1.4) reported earlier. The maximum relative error of this approximation is not quite as good as for formula (4.13), but is still impressive: at $c / l=0.75$ the maximum relative error is around $2 \%$ (about the same as for (4.13)) and for $c / l=0.9$ it is around $12 \%$ (slightly worse). The surprisingly wide range of no-shear fractions for which these formulae give good accuracy arguably obviates the need for higher-order approximations, but these are easily derivable in principle.

\section{Verification against previous results}

When formally expanded in powers of the no-shear fraction our formulae crosscheck with other known results. First, Philip (1972) has shown that for a flat meniscus 


\section{G. Crowdy}

with $\theta=0, c=1$ and at any no-shear fraction,

$$
\lambda=\frac{2 l}{\pi} \log \sec \left[\frac{\pi}{2 l}\right]=-\frac{1}{\delta} \log \cos \delta \approx \delta\left[\frac{1}{2}+\frac{\delta^{2}}{12}+\frac{\delta^{4}}{45}+\cdots\right], \quad \delta \equiv \frac{\pi}{2 l} .
$$

But for $\theta=0$ we find $\alpha(0)=1 / 2$ and $\beta(0)=1 / 8$ and it can be verified that (1.4) agrees with (5.1) to this order of expansion in $\delta$.

By taking their microchannel width to infinity, Sbragaglia \& Prosperetti (2007) performed an analytical study for small protrusion angles $\theta$ in a semi-infinite domain. The expansion of (4.15) for small $\delta$ is

$$
\frac{\lambda_{2}}{c}=\delta c \alpha(\theta)\left[1+\frac{c^{2} \delta^{2} \alpha(\theta)}{3}+\delta^{4} c^{4}\left(\frac{2 \beta(\theta)}{15}+\frac{\alpha(\theta)^{2}}{9}\right)+\cdots\right] .
$$

A Taylor expansion of $\alpha(\theta)$ and $\beta(\theta)$ to linear order in the protrusion angle $\theta$ gives

$$
\alpha(\theta)=\frac{1}{2}+\frac{\theta}{3 \pi}+\cdots, \quad \beta(\theta)=\frac{1}{8}+\frac{\theta}{30 \pi}+\cdots .
$$

On substitution of (5.3) into (5.2), we find

$$
\frac{\lambda_{2}}{c}=\delta c\left[\frac{1}{2}+\frac{\delta^{2} c^{2}}{12}+\frac{\delta^{4} c^{4}}{45}+\cdots\right]+\delta c\left(\frac{\theta}{\pi}\right)\left[\frac{1}{3}+\frac{c^{2} \delta^{2}}{9}+\frac{24}{675} c^{4} \delta^{4}+\cdots\right]+\cdots
$$

The first term agrees with (5.1), valid for $\theta=0$, once we set $c=1$. On setting $\delta=\pi \xi / 2 c$ the normalized leading-order correction due to the meniscus curvature is

$$
\frac{c \theta \xi}{2}\left[\frac{1}{3}+\frac{\xi^{2} \pi^{2}}{36}+\frac{\xi^{4} \pi^{4}}{450}\right]=-\frac{c^{2}}{2 R}\left[\frac{\xi}{3}+\frac{\xi^{3} \pi^{2}}{36}+\frac{\xi^{4} \pi^{4} c^{4}}{450}\right]
$$

where, for small meniscus deflection downwards into the grooves, $\theta \approx \sin \theta=-c / R$. We thus retrieve the result in (45)-(47) of Sbragaglia \& Prosperetti (2007), who derived it using quite different techniques. In summary, various series expansions of our formulae (1.3) and (4.13), or (1.4), give results that are consistent with other studies. Note, however, that we continue to present (1.3) and (1.4) in the unexpanded form arising naturally from our analysis.

\section{A reciprocity result}

In view of their convenient explicit forms we expect that the slip length formulae (1.3) and (1.4), and the associated complex potentials (3.1) and (4.4), will be useful in a variety of studies of superhydrophobic surfaces where, for example, additional physical effects are included (such as heat transfer, Marangoni or thermocapillary effects, or the influence of an enclosed gas phase). We end by showing how to combine the new formulae with a useful reciprocity result based on one of Green's identities.

Let $w_{2}(z)=\operatorname{Im}\left[\dot{\gamma} h_{2}(z)\right]$, with associated slip length $\lambda_{2}$. Suppose $\hat{w}$ is the solution of the same problem of shear flow over the bubble mattress with all the same boundary conditions imposed on $w_{2}(z)$ except on the meniscus, where we now require

$$
\frac{\partial \hat{w}}{\partial n}=\mathscr{A}(z, \bar{z}),
$$




\section{Analytical formulae for longitudinal slip lengths}

where $\mathscr{A}(z, \bar{z})$ is some specified function (derived, say, from inclusion of additional physical effects). Let the slip length for this modified problem be $\hat{\lambda}$. By Green's identity, the harmonicity of $w_{2}$ and $\hat{w}$, and the divergence theorem, we deduce that

$$
0=\int_{D}\left[w_{2} \nabla^{2} \hat{w}-\hat{w} \nabla^{2} w_{2}\right] \mathrm{d} A=\oint_{\partial D} w_{2} \frac{\partial \hat{w}}{\partial n} \mathrm{~d} s-\oint_{\partial D} \hat{w} \frac{\partial w_{2}}{\partial n} \mathrm{~d} s,
$$

where $\partial D$ is the boundary of $D$. On the edges of the period window for which $|z| \geqslant l$,

$$
w_{2}=\dot{\gamma}\left(y+\lambda_{2}\right)+O\left(\delta^{5}\right), \quad \frac{\partial w_{2}}{\partial n} \mathrm{~d} s= \pm \frac{\partial w_{2}}{\partial x} \mathrm{~d} y,
$$

where we have used (4.7). Hence the contributions to the right-hand side of (6.2) from the side edges are small (for small $\delta$ ) while the no-slip portions of the surface do not contribute at all. We then arrive at the approximation

$$
\hat{\lambda} \approx \lambda_{2}-\frac{1}{2 l} \int_{\text {meniscus }} \mathscr{A}(z, \bar{z}) \operatorname{Im}\left[h_{2}(z)\right] \mathrm{d} s,
$$

where we have integrated around $\partial D$ in an anticlockwise direction and used the farfield conditions. By virtue of the results of this paper the right-hand side of (6.4) is an integral expression for the required slip length $\hat{\lambda}$ accurate to the same order in the no-shear fraction as the solution $h_{2}(z)$ and $\lambda_{2}$ used to obtain it.

\section{Discussion}

An asymptotic analysis similar to that presented here is, in principle, possible to generalize the dilute approximation to the transverse slip length over this class of surfaces found by Davis \& Lauga (2009); the biharmonic nature of the field equations there render the technical details more challenging. But complementary results in this direction would provide a fuller analytical description of the so-called slip tensor (Bazant \& Vinogradova 2008; Asmolov \& Vinogradova 2012) for these surfaces, thereby generalizing the fairly complete analytical description in the dilute limit already available on combining the analytical formulae of Davis \& Lauga (2009) and Crowdy (2010).

The study here has imposed idealized shear-free boundary conditions at the liquid-gas interfaces and ignores additional dissipation associated with an enclosed gas. Incorporating dissipation in the gas subphase is a topic of much recent research: Schönecker \& Hardt (2013) and Schönecker, Baier \& Hardt (2014) have proposed a semianalytical method that approximates the liquid-gas interface as a constant shear-stress boundary (leading to a non-uniform local slip length); Nizkaya, Asmolov \& Vinogradova (2014) have put forward a general 'gas cushion model' based on an operator method. Those authors have executed detailed studies of their models in the case of unidirectional (one-dimensional) surfaces with flat interfaces. Our results here - especially coupled with the reciprocity result of $\S 6-$ might well be useful in extending those investigations to the important case of curved menisci.

\section{Acknowledgements}

This work is supported by EPSRC Fellowship EP/K019430/1, a Royal Society Wolfson Research Merit Award and EPSRC grant EP/K041134/1. The author is grateful to the authors of Teo \& Khoo (2010) for providing comparison data. 


\section{G. Crowdy}

\section{References}

Asmolov, E. S. \& Vinogradova, O. I. 2012 Effective slip boundary conditions for arbitrary one-dimensional surfaces. J. Fluid Mech. 706, 108-117.

Bazant, M. Z. \& Vinogradova, O. I. 2008 Tensorial hydrodynamic slip. J. Fluid Mech. 613, $125-134$.

Crowdy, D. G. 2010 Slip length for longitudinal shear flow over a dilute periodic mattress of protruding bubbles. Phys. Fluids 22, 121703.

Davis, A. M. J. \& LAUGA, E. 2009 Geometric transition in friction for flow over a bubble mattress. Phys. Fluids 21, 011701.

NG, C.-O. \& WANG, C. Y. 2011 Effective slip for Stokes flow over a surface patterned with twoor three-dimensional protrusions. Fluid Dyn. Res. 43, 065504.

Nizkaya, T. V., Asmolov, E. S. \& Vinogradova, O. I. 2014 Gas cushion model and hydrodynamic boundary conditions for superhydrophobic textures. Phys. Rev. E 90, 043017.

PhILIP, J. R. 1972 Flows satisfying mixed no-slip and no-shear conditions. Z. Angew. Math. Phys. 23, 353-372.

Rothstein, J. P. 2010 Slip on superhydrophobic surfaces. Annu. Rev. Fluid Mech. 42, 89-109.

Sbragaglia, M. \& Prosperetti, A. 2007 A note on the effective slip properties for microchannel flows with ultrahydrophobic surfaces. Phys. Fluids 19, 043603.

Schönecker, C., BAIER, T. \& HARDT, S. 2014 Influence of the enclosed fluid on the flow over a microstructured surface in the Cassie state. J. Fluid Mech. 740, 168-195.

SCHÖNECKER, C. \& HARDT, S. 2013 Longitudinal and transverse flow over a cavity containing a second immiscible fluid. J. Fluid Mech. 717, 376-394.

Steinberger, A., Cottin-Bizonne, C., Kleimann, P. \& Charlaix, E. 2007 High friction on a bubble mattress. Nat. Mater. 6, 665-668.

TeO, C. J. \& KHOо, B. C. 2010 Flow past superhydrophobic surfaces containing longitudinal grooves: effects of interface curvature. Microfluid. Nanofluid. 9, 499-511. 